\title{
Cardiovascular Reactivity in Hypertensive Rats
}

\author{
Fumio Shibayama, B.P., * Susumu Mrzogami, B. P., * \\ and Hirofumi Sokabe, M.D.
}

\begin{abstract}
SUMmARY
Cardiovascular (CV) reactivity to norepinephrine (NE) and angiotensin II (Ang) was determined in the spontaneously hypertensive rat (SHR), and in the rat made hypertensive by clipping the renal artery and contralateral nephrectomy, or by deoxycorticosterone and salt administration. The full dose-response curves were obtained without anesthesia after pithing, decerebration, and vagotomy. CV reactivity to Ang was increased in the SHR, but only increased slightly in hypertension by clipping. This change in CV reactivity does not explain existent high blood pressure, since Ang content of the blood in the hypertensive states is not always increased. CV reactivity to NE was essentially unchanged in the 3 types of hypertension. The results indicate that the observed high blood pressure is not due to an increased sensitivity to NE in the hypertensive rats, and that NE must be available in a larger amount at the receptor sites in the cardiovascular system to maintain the higher blood pressure.
\end{abstract}

\section{Additional Indexing Words :}

Angiotensin II Norepinephrine Sympathetic nervous system

EURAL innervation of the cardiovascular system is sympathetic and 1 parasympathetic to the heart, and only sympathetic to the peripheral blood vessels except very special parts. Elimination of the neural innervation by destroying the central nervous system (CNS) lowers blood pressure to a basal level in normotensive and hypertensive animals. ${ }^{1-4)}$ The results have been interpreted that (i) blood pressure is maintained by the autonomic, particularly sympathetic, nervous system in the normal state, (ii) high blood pressure is also maintained by the sympathetic nervous system in the hypertensive state, and (iii) high blood pressure is not mediated with a humoral pressor factor.

Determination of the cardiovascular (CV) reactivity to norepinephrine, chemical transmitter of the sympathetic nervous systcm, in the hypertensive animals after GNS destruction would furnish the following basic informations

From the Department of Pharmacology, Toho University School of Medicine, Tokyo.

* Present Address: the Iyakushigen Institute for Medical Research, Tokyo.

Received for publication June 26, 1970. 
on the mechanism of hypertension ${ }^{5)}$ : (i) if $\mathrm{GV}$ reactivity were increased, the increased sensitivity of the cardiovascular system to norepinephrine would explain hypertension, and (ii) if reactivity were not increased, high blood pressure must be explained by an increased activity in the sympathetic nervous system.

In order to obtain a reliable result, it is necessary to: (i) avoid the vertical bias by obtaining the full dose-response curve, (ii) exclude the effects of anesthetics to the cardiovascular system, (iii) eliminate CNS completely, and (iv) study the reactivity at the same blood pressure levels in the hypertensive and normotensive animals. We have devised a preparation of the rat which meets these requirements by pithing, decerebration, and vagotomy, and obtained full dose-response curves.

The CV reactivity to norepinephrine (NE) and angiotensin II (Ang) was obtained in the above described preparation of spontaneous, renal, and adrenal hypertension. Ang alone could not explain the mechanism of either type of hypertension. CV reactivity to NE was essentially the same in hypertensive and normotensive animals. The results indicate that (i) the observed high blood pressure in the hypertensive rats is not due to an increased sensitivity to NE, (ii) an increased activity in the sympathetic nervous system must be present in the hypertensive animals, and consequently (iii) NE must be available in a larger amount at the sympathetic receptors in the cardiovascular system to maintain the higher blood pressure.

\section{Methods}

Spontaneously hypertensive rats (SHR), ${ }^{6}$ renal hypertension induced by clipping, and DCA hypertension were studied. Male rats were used throughout the experiments. SHR were F12-17 furnished at Fll by the Department of Pharmacology, Toho University School of Medicine to Iyakushigen Institute for Medical Research. They were 17 to 23 weeks of age, weighing 270 to $400 \mathrm{Gm}$. Wistar strain of the Animal Center Laboratory, Kyoto University Faculty of Medicine, from which the SHR had been separated, used as controls, were 18 to 23 weeks of age, weighing 290 to $380 \mathrm{Gm}$. Renal hypertension was induced by clipping the left renal artery with a silver ribbon and removing the right kidney a week later in rats of Donryu strain, 8 to 9 weeks of age, weighing 200 to $230 \mathrm{Gm}$. They were used 4 to 8 weeks after clipping. Body wt ranged 220 to $320 \mathrm{Gm}$. DCA hypertension was induced by injecting deoxycorticosterone acetate $(1 \mathrm{mg} . \times 3 / \mathrm{wk}, \mathrm{sc})$ in Donryu strain unilaterally nephrectomized a week previously and given $1 \%$ saline instead of drinking water. They were 8 to 9 weeks of age, weighing 200 to $230 \mathrm{Gm}$. After treatment for 4 to 6 weeks the rats, weighing 240 to $310 \mathrm{Gm}$. were used for the study. Systolic blood pressures were measured by the plethysmographic tail method. Normal rats of Donryu strain, matched in age and body wt were used as controls. 
Dose-blood pressure-response curves to NE and Ang were obtained in a preparation in which the central nervous system was eliminated by pithing, decerebration, and vagotomy. The procedures used was a modification of the method reported previously. ${ }^{7)}$ Under ether anesthesia the femoral artery was cannulated, and the mean systemic blood pressure was recorded with a electronic system (Nihon Kohden MP-4T, RP-2, RM-20) after 120 min. when anesthesia had been completely withdrawn. The rat was again anesthetized with ether, and the trachea and femoral vein were cannulated. The tracheal cannula was connected to a positive-pressure respirator (Natume $\mathrm{KN}-56$ ). The rat was pithed by inserting a steel rod through the orbital fossa to the end of spinal canal. Cerebral tissue was further destroyed by cotton balls pressed into the cranial cavity through an opening on the cranium. The vagosympathetic trunks were cut bilaterally at the level of tracheal cannulation. The rats were then heparinized (200 units/Kg., iv) and used for the determination of $\mathrm{CV}$ reactivity.

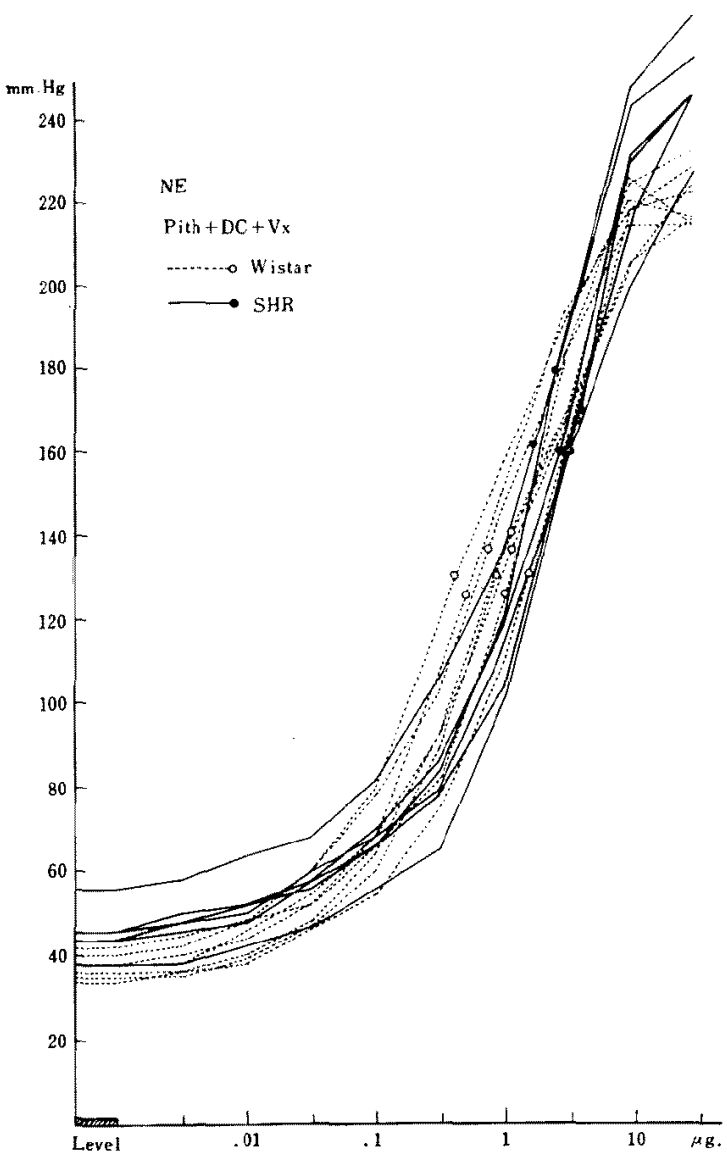

Fig. 1. GV reactivity to NE in the spontaneously hypertensive rat (SHR) and normal Wistar strain. Each composition of connected straight lines (DR curve) represents 1 rat. Level indicates blood pressure level after CNS destruction. Blood pressure before CNS destruction is plotled on the DR curve. 
A series of NE or Ang solutions, diluted at a constant ratio, were injected intravenously in an ascending order in concentration. The substances were not injected on per body wt basis, but in net amounts, because the dose ranges were far greater than the body wt range. The maximum rise in blood pressure was taken as the response. The substances were dissolved in $0.9 \%$ saline to a volume of $0.1 \mathrm{ml}$. Immediately after the injection the venous cannula was flushed with $0.05 \mathrm{ml}$. of saline. dl-Norepinephrine bitartrate and synthetic $\mathrm{Asn}^{1}-\mathrm{Val}{ }^{5}$-angiotensin II were used. Doses of NE referred to one half of the free base.

\section{Results}

\section{Cardiovascular reactivity to norepinephrine}

Dose-response (DR) curves of SHR and normal Wistar rats to NE of 9 doses, 0.003-30 $\mu$ g., with blood pressure levels before and after destruction

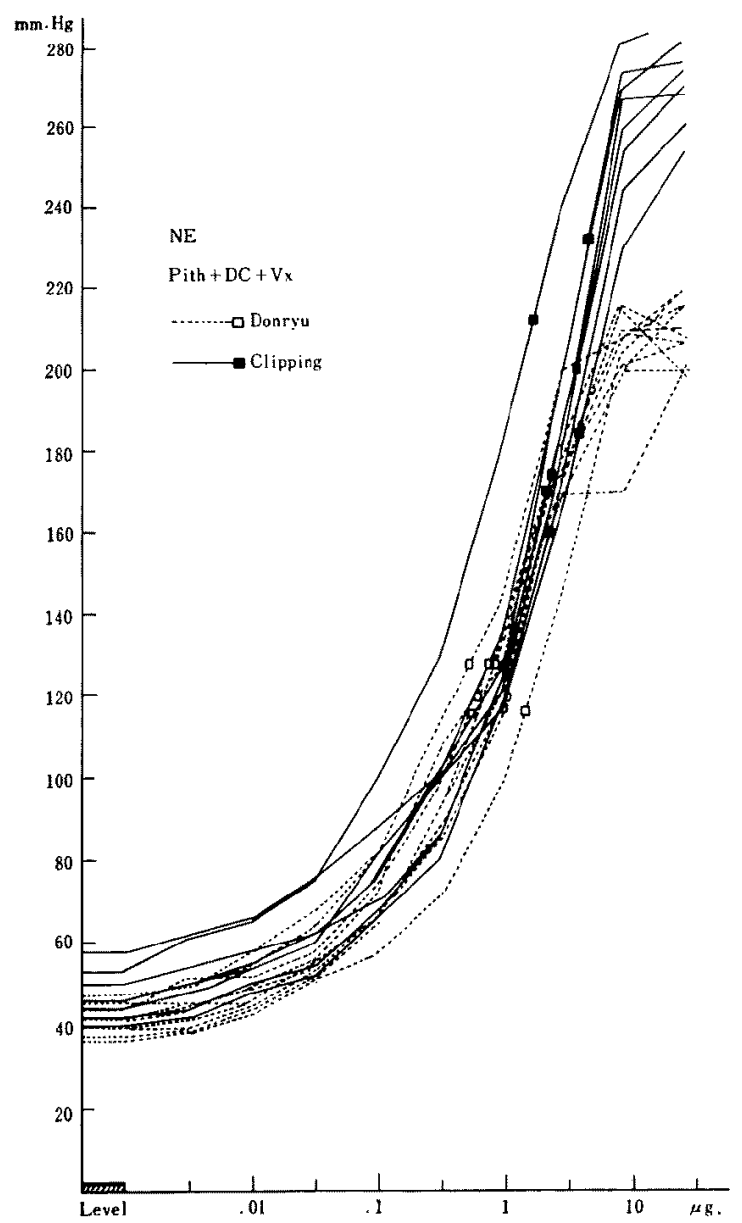

Fig. 2. CV reactivity to NE hypertension induced by clipping and in the normal Donryu rat. 
of the central nervous system (CNS destruction) are shown in Fig. 1. The results in hypertensive rats induced by clipping or DCA and in normal rats of Donryu strain are given in Figs. 2 and 3.

DR curves to NE of SHR showed the same course as those of Wistar rats, although they reached higer levels at the largest 2 doses in hypertensive rats than in the controls. High blood pressure levels of SHR correspond to 2-4 $\mu \mathrm{g}$. NE on the DR curves. Normal blood pressure levels of Wistar rats correspond to $0.4-2 \mu \mathrm{g}$. NE.

Essentially the same results were obtained in hypertensive rats induced by clipping or DCA.

Cardiovascular reactivity to angiotensin II

DR curves of SHR and normal Wistar rats to Ang of 8 doses, 0.003-

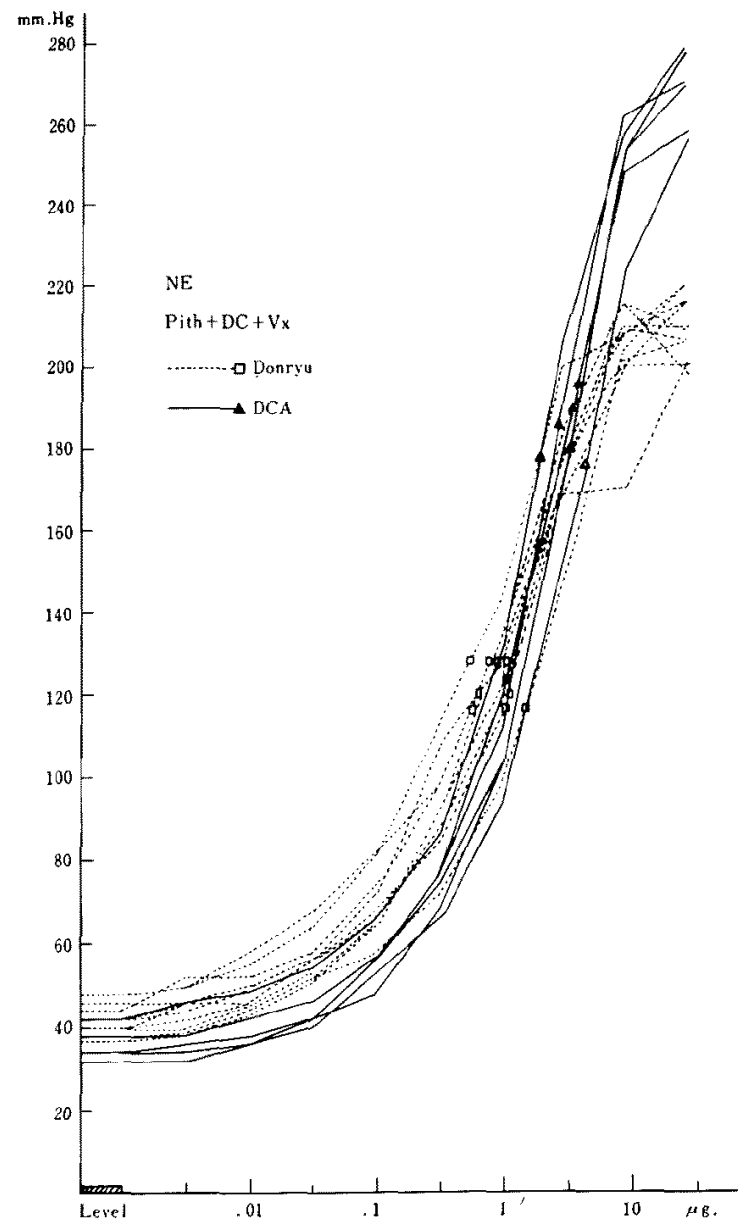

Fig. 3. CV reactivity to NE in DCA hypertension and in the normal Donryu rat. 
$10 \mu \mathrm{g}$., with blood pressure levels before and after CNS destruction are shown in Fig. 4. The results in hypertensive rats induced by clipping or DCA and the controls are given in Figs. 5 and 6.

The DR curves to Ang of SHR were shifted to upper left. CV reactivity was increased to Ang. There was slight supersensitivity in hypertension induced by clipping. The high blood pressure levels of some hypertensive rats did not correspond to those obtained by any dose of Ang in the DR curves.

DR curves followed almost the same course in DCA hypertension and the control. High blood pressure levels of DCA hypertensive rats with I exception were higher than any points of DR curve reached by Ang injection.

Blood pressure levels after CNS destruction

After CNS destruction blood pressure fell to a basal level, 28 to $56 \mathrm{~mm}$. $\mathrm{Hg}$, as reported previously.1-4) Table I summarizes the results and their statistical analyses. The levels were higher in SHR and hypertension induced by clipping.

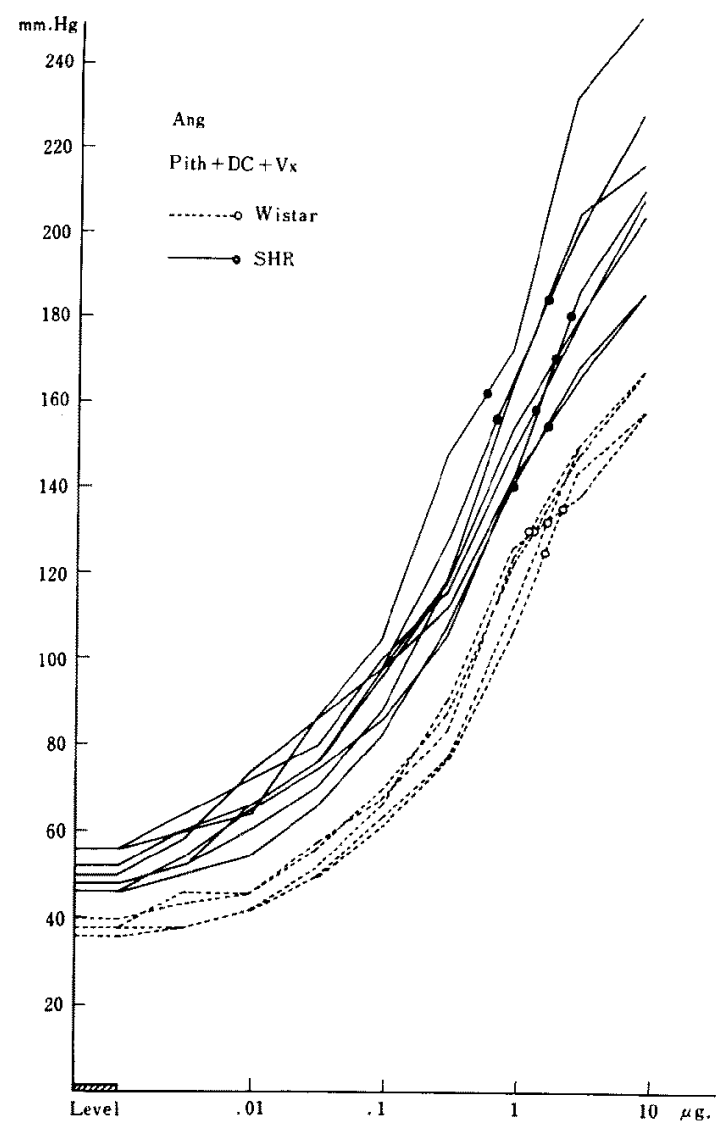

Fig. 4. CV reactivity to Ang in the spontaneously hypertensive rat (SHR) and in the normal Wistar strain. 
It was lower in DCA hypertension. Although the differences are statistically significant, they are small, and can not account for the observed high blood pressures. The basal levels may be determined by humoral and myogenic factors acting on the cardiovascular system.

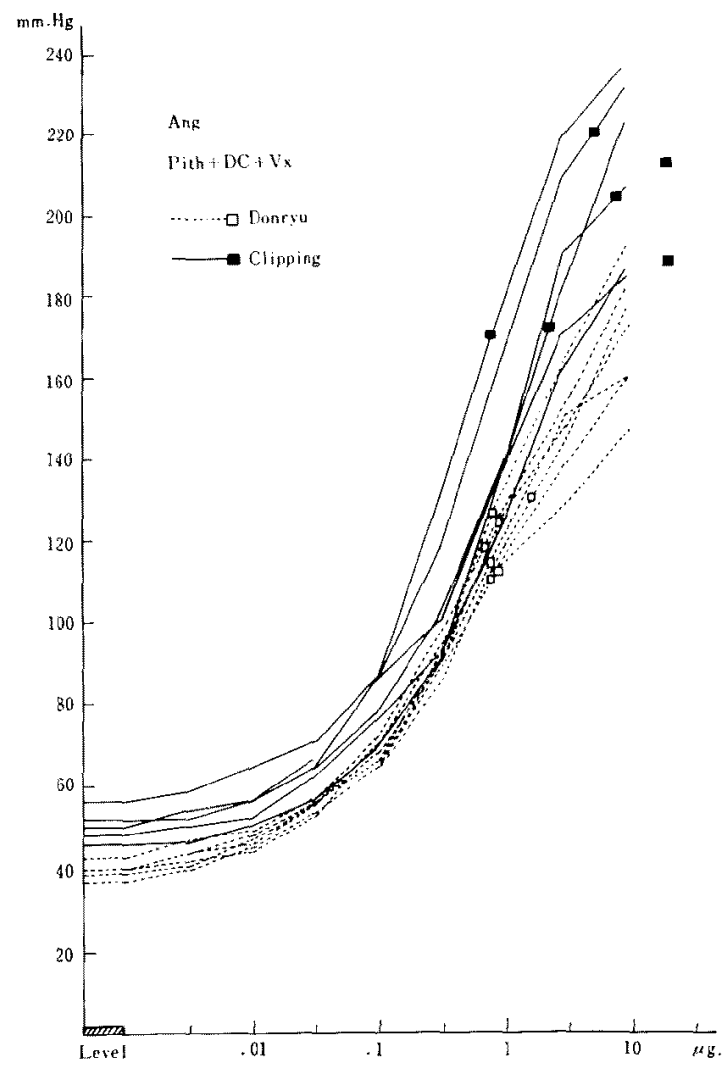

Fig. 5. CV reactivity to Ang in hypertension induced by clipping and in the normal Donryu rat.

Table I. Blood Pressure Levels After CNS Destruction in Hypertensive Rats

\begin{tabular}{l|c|c|c}
\hline & No. of Rats & BP (mm.Hg) & P \\
\hline Wistar & 13 & $37.9 \pm 0.6^{*}$ & \\
SHR & 14 & $48.3 \pm 1.4$ & $0.01>$ \\
Donryu & 18 & $41.3 \pm 0.8$ & \\
Clipping & 14 & $48.5 \pm 1.4$ & $0.01>$ \\
DCA & 16 & $35.1 \pm 1.3$ & $0.01>$
\end{tabular}

* mean $\pm S E$ 


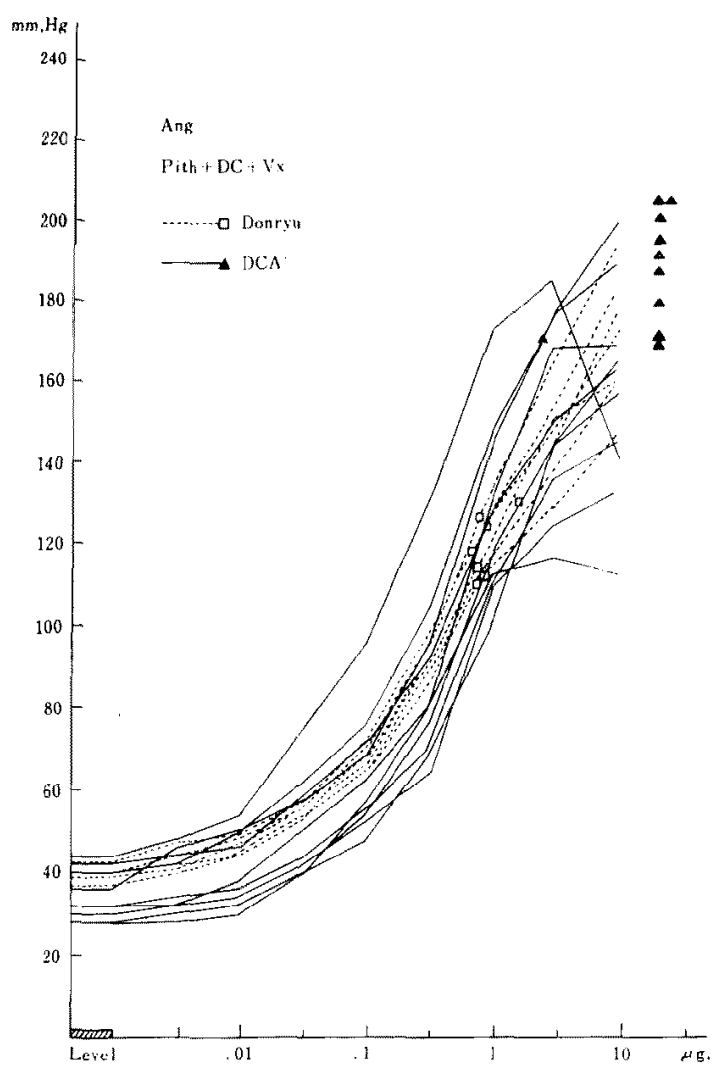

Fig. 6. CV reactivity to Ang in DCA hypertension and in the normal Donryu rat.

\section{Discussion}

Cardiovascular (GV) reactivity has been defined as the degree with which the heart and peripheral vascular system respond to quantitated stimuli. ${ }^{8}$ ) A number of results have been conflicting both clinically and experimentally. ${ }^{5), 81-10)}$ Recent studies using the aortic strip or isolated atria have not clarified the problem. ${ }^{111-13}$

Difficulties are in methodology. Several conditions described in the introduction must be satisfied to botain pertinent results for considering the mechanism of hypertension. ${ }^{5}$ Our preparation of the rat fits these requirements. CNS destruction was so complete that all their blood supply was eliminated, and that absorption of the destroyed materials into the circulation would not occur. The blood pressure levels were stable during the experiments, and the rats were tolerated large amounts of NE or Ang.

$\mathrm{CV}$ reactivity to $\mathrm{NE}$ was essentially the same in the 3 types of hyper- 
tension used in this study and in the controls. A dose of NE elicited the same blood pressure rise from the basal level after CNS destruction in either condition, except that the responses reached higher levels at the largest 2 doses in the hypertensive rats. This was probably due that the hypertensive heart and blood vessels had been adapted to the higher blood pressure level. To maintain hypertensive levels the amount of NE corresponding to an intravenous dose of $2-4 \mu \mathrm{g}$. must be available at the sympathetic receptors in hypertensive rats. It was $0.4-2 \mu \mathrm{g}$. in the controls. On intravenous injections the concentration of NE in blood is abnormally higher than is obtained by endogenous transmitter released from the nerve endings, but the amount available to the receptor sites determines the effect. The results indicate that the elevated blood pressure in hypertensive rats is maintained by the sympathetic nervous system, the activity of which is increased presynaptically, but not postsynaptically.

Various studies reported increased sensitivities to $\mathrm{NE}$ in hypertensive states. ${ }^{14)-18)}$ However, they utilized methods not to satisfy the conditions indicated above: they determined the reactivity in preparations without CNS destruction, ${ }^{14)}$ of certain denervated vascular bed, ${ }^{15)-18}$ ) with artificial solutions to perfuse, ${ }^{17), 18)}$ or without equalizing the blood pressure levels. ${ }^{14)-16)}$ Sympathetic postganglionic neurons were intact in our preparation, and their uptake mechanism should be preserved.

The release and reuptake of the transmitter is very rapid, and movement of $\mathrm{NE}$ in larger amounts in hypertension might not influence catecholamine metabolism as determined by urinary excretion. ${ }^{19)}$ De Champlain et al. found a defect in the storage and retention of NE by the granules of the sympathetic nerves of hypertensive rats, and suggested that amounts of physiologically active catecholamine available at the receptor are increased in hypertension. ${ }^{20}$ ) Recent studies by more elaborated methods also indicated increased turnover of NE in peripheral organs of hypertensive rats. ${ }^{21)}$,2) There are other evidences compatible with this view. ${ }^{23), 24)}$

The pathogenesis of hypertensive cardiovascular disease is not established. Nor is it certain that there is common mechanism responsible for various types of experimental hypertension. However, our results indicate that the elevated blood pressure may be maintained by increased activity of the sympathetic nervous system commonly in 3 types of hypertensive rats, although the detailed mechanism causing this increased activity by heredity, clipping, or deoxycorticosterone injection was unknown.

Our results furnished a basic information on the mechanism of hypertension: the cardiovascular system is not supersensitive to NE in hypertension. The fact must always be considered with the increased catecholamine metab- 
olism in hypertension, for which evidences have been accumulating. ${ }^{20)-22)}$ If the cardiovascular system were subsensitive to NE, the increased catecholamine metabolism would not elicit hypertension. If it were already supersensitive, the increase might mean nothing.

The preliminary report that hypertension induced by clipping became superexcitable to $\mathrm{NE}^{25}$ ) was not correct. Sensitivity to NE of normal Donryu rats was abnormally low at that time perhaps due to an inferior care of animals, which was confirmed in 2 series of experiments other than the control included in this paper.

CV reactivity to Ang was increased definitely in SHR, and slightly in hypertension induced by clipping. Increased sensitivity to Ang could not explain the hypertension in SHR or by clipping, because Ang concentration in blood would not be as high as that used in our experiments to obtain DR curves. Blood pressure levels sometimes exceeded that of the DR curves. This constitutes further evidence against the renin-angiotensin hypothesis as the cause of hypertension.

\section{RefERENCES}

1. Taquini, A. C., Jr., Blaquier, P. C., and Bohr, D. F.: Am. J. Physiol. 201: 1173, 1961.

2. Taquini, A. C., Jr.: Arch. Int. Pharmacodyn. 140: 556, 1962.

3. Taquini, A. C., Jr.: Circulat. Res. 12: 562, 1963.

4. Okamoto, K., Nosaka, S., Yamori, Y., and Matsumoto, M.: Jap. Heart J. 8: 168, 1967.

5. Sokabe, H.: Experimental Hypertension. An Introductory Textbook. Eikodo, Tokyo, p. 97, 1968 (in Japanese).

6. Okamoto, K. and Aoki, K.: Jap. Circulat. J. 28: 943, 1963.

7. Sokabe, H., Shibayama, F., Mizogami, S., and Sakai, F.: Jap. Heart J. 3: 233, 1965.

8. Page, I. H., Kaneko, Y., and McCubbin, J. W.: Circulat. Res. 18: 171, 1966.

9. Mendlowitz, M.: Am. Heart J. 73: 121, 1967.

10. Khairallah, P. A. (ed.): In Renal Hypertension, edited by I. H. Page and J. W. McCubbin. Year Book Medical, Chicago, p. 160, 1968.

11. Wohl, A. J., Hausler, L. M., and Roth, F. E.: J. Pharm. Exp. Therap. 162: 109, 1968.

12. Spector, S., Fleisch, J. H., Maling, H. M., and Brodie, B. B.: Science 166: 1300, 1969.

13. Baum, T.: J. Pharm. Exp. Therap. 165: 176, 1969.

14. Okamoto, K., Hazama, F., Takeda, T., Tabei, R., Nosaka, S., Fukushima, M., Yamori, Y., Matsumoto, M., Haebara, H., Ichijima, K., and Suzuki, Y.: Jap. Circulat. J. 30: 987, 1966.

15. Baum, T. and Shropshire, A. T.: Am. J. Physiol. 212: 1020, 1967.

16. Baum, T. and Shropshire, A. T.: Am. J. Physiol. 213: 499, 1967.

17. Haeusler, G. and Haefely, W.: Naunyn-Schmiedeberg's Arch. Pharmakol, 266: 18, 1970.

18. Folkow, B., Hallbäck, M., Lundgren, Y., and Weiss, L.: Acta Physiol. Scand. 79: 373, 1970.

19. De Quattro, V. and Sjoerdsma, A.: J. Clin. Invest. 49: 2359, 1968.

20. De Champlain, J., Krakoff, L., and Axelrod, J.: Circulat. Res. 24-25: I-75, 1969.

21. Nakamura, K., Gerold, M., and Thoenen, H.: Naunyn-Schmiedeberg's Arch. Pharmakol. (in press).

22. Yamori, X., Lovenberg, W. M., and Sjoerdsma, A.: In Proceedings of 6 th Annual Scientific Meeting of the Council for the Spontaneously Hypertensive Rat, Chiba 1970. 
23. Zimmerman, B. G., Rolewicz, T. F., Donham, E. W., and Gisslen, J. L.: Am. J. Physiol. 217: $798,1969$.

24. Baum, T. and Shropshire, A. T.: Arch. Int. Pharmacodyn, 181 : 405, 1969.

25. Shibayama, F., Mizogami, S., and Sokabe, H.: Nihon Taishitsu Shi 30: 161, 1966 (in Japanese). 